\title{
Comprehensive management of haemophilia A and B
}

G N Lucas ${ }^{1}$

Sri Lanka Journal of Child Health, 2002; 31: 34-45

(Key words: haemophilia, management)

Management of haemophilia involves recognition and treatment of acute haemorrhagic episodes, institution of preventive measures against bleeding, optimisation of conditions under which treatment is administered, genetic counselling and therapy of the musculoskeletal and central nervous system (CNS) complications of the disease ${ }^{1}$. All patients with haemophilia should ideally be followed periodically in a large multidisciplinary haemophilia centre ${ }^{1,5}$.

\section{Centralised care}

Comprehensive management of haemophilia implies a coordinated programme to maintain and restore physical, psychological and social wellbeing ${ }^{1}$. An individual paediatrician would find it difficult to provide such care whereas treatment teams at haemophilia centres can deliver competent, thorough care efficiently to large numbers of patients ${ }^{1}$. During the past 4 decades, haemophilia care has become more centralised. This trend has been aided in some countries by directives and grants to form centres ${ }^{1}$.

Centralised care was developed because severe haemophilia creates multiple problems. Firstly, the patient suffers recurrent acute haemorrhages that may disrupt school attendance. Secondly, he has to contend with chronic problems such as musculoskeletal disability and discomfort, a low level of self-esteem and so on. In addition, concurrent unrelated illnesses often are complicated by the bleeding disorder. Tonsillitis, for example, is often complicated by bleeding into the mouth or into the retropharyngeal area ${ }^{1}$.

Comprehensive management requires the cooperation of a team of care-givers such as paediatricians, haematologists, orthopaedic surgeons, nurses, physiotherapists, dentists, genetic counsellors, psychologists, social workers and vocational counsellors ${ }^{1}$. Patients living near a centre can receive all medical care there. Those living at a distance may depend on a local doctor for routine care and travel to the centre for periodic examinations and consultations. A major function of a centre is to offer advice to outlying

${ }^{1}$ Consultant Paediatrician. practitioners and to receive patients with problems too complex for effective local treatment ${ }^{1}$.

There are some negative aspects of centralisation. Patients with haemophilia become isolated from the general medical community so that many paediatricians will regard haemophilia as a rare disease and have little idea of its manifestations or treatment. Furthermore, changes in government funding policy can occur abruptly and an institution serving patients with an expensive disease can founder ${ }^{1}$.

\section{Preventive care}

Newborn infants, in whom haemophilia is a distinct possibility, should not be circumcised until exclusion of the diagnosis. Blood for investigations should be obtained from superficial veins and pressure should be applied subsequently until bleeding stops. Femoral or jugular venepuncture is contraindicated as leakage could cause a large haematoma in a dangerous location ${ }^{1}$.

Haemophiliacs should receive the routine immunisations in infancy including immunisation against hepatitis B. The small-gauge needles used for these injections rarely cause haematomata. Standard preventive dental supervision is mandatory ${ }^{1}$.

Parents need counselling to cope with the diagnosis of haemophilia in their baby. Mothers may feel they are to blame for transmitting the defective gene and compensate by overprotecting the child and giving him too much attention. Fathers and siblings may then feel excluded. Both parents should be urged to participate in the child's upbringing and haemophilia management. Parental needs are well described by Agle et $\mathrm{a}^{12}$.

Parents need to know how to arrange reasonably safe play with other children so that the boy can experience normal social growth. Some degree of physical protection can be given by such measures as carpeting bare floors, padding the crib and playpen and padding long-sleeved shirts and long trousers on the inside, at the levels of the elbows 
and knees. Passenger restraints in cars are doubly important for a child with haemophilia ${ }^{1}$.

Sensible physical exercise is encouraged because strong muscles help support joints and decrease the frequency of haemorrhage. Ideal exercises strengthen muscles and preserve normal joint motion without increasing the force of gravity on the joints. Swimming and cycling are encouraged but jogging and contact sports are discouraged. A set of exercises, preferably where the whole family joins in, can be performed daily at home. Exercise is the cheapest, but most ignored, form of prophylactic care for haemophilia ${ }^{3}$.

Thorough education of the parents and child regarding the nature of haemophilia and its management enables the family to cooperate appropriately in the boy's care. Parents tolerate genetic counselling offered with a neutral attitude but may resist and resent a counsellor who advises restriction of reproduction ${ }^{1}$.

Factor VIII or IX assays should be done on female relatives who may be carriers of haemophilia, for some carriers have factor levels below the haemostatic range. They should be warned of the possibility of excessive bleeding from trauma or surgical operations and told how to obtain proper care $^{1}$. Tests of appropriate male relatives of patients with mild sex-linked haemophilia may uncover some hitherto undiagnosed mild haemophiliacs ${ }^{1}$.

Education of the boy about haemophilia should take place throughout the growing years. If his parents have been administering his clotting factor at home, he should be ready to take over that responsibility as a teenager so that he progresses normally towards adult independence. Boys with haemophilia usually attend schools designed for normal children. Teachers need information about the nature and management of medical problems that may arise at school ${ }^{1}$.

\section{Replacement therapy}

When bleeding episodes occur, replacement therapy is essential to prevent pain, disability or life-threatening haemorrhage. The aim of therapy is to increase factor VIII or IX activity in the plasma to a level that secures haemostasis. This can be done by the intravenous (IV) infusion of fresh frozen plasma (FFP) or of factor VIII or IX concentrates ${ }^{4}$. Today, it is frequently the cost, rather than the quality or safety of clotting factor, that restricts implementation of optimal therapeutic strategies for haemophilia ${ }^{4}$.

The "two commandments" of acute management of haemophilic bleeding are ${ }^{5}$ :
1. Believe the patient and/or his parent; if he thinks he is bleeding, then he should be treated even though physical signs are not evident.

2. If in doubt, infuse. Over-treatment of a sprain may occasionally result, but an untreated haemarthrosis may result in permanent damage.

The dosing regimen for factor replacement in haemophilia is based on 3 important pharmacologic and therapeutic principles ${ }^{4}$ :

1. The volume of the clotting factor distribution within intravascular or extravascular compartments which affects in vivo factor recovery in plasma following an infusion.

2. The factor survival, or half-disappearance time, in plasma.

3. The minimal haemostatic factor level required to control the particular type and extent of the haemorrhage.

Furthermore, clotting factor is dosed in "units" of activity, with 1 unit of factor representing the amount present in $1 \mathrm{ml}$ of normal plasma ${ }^{4}$.

One unit of factor VIII raises the plasma level by approximately $0.02 \mathrm{U} / \mathrm{ml} \quad(2 \%)$. Its halfdisappearance time from circulation is biphasic, averaging approximately 12 hours. On the other hand, one unit of factor IX, which has a larger volume of distribution, raises plasma levels by 0.01 $\mathrm{U} / \mathrm{ml}(1 \%)$. Factor IX has a plasma halfdisappearance time of approximately 24 hours ${ }^{4}$.

The minimum haemostatic plasma factor levels are determined according to the type and extent of bleeding. In general, 30-50\% of normal factor levels are required for mild bleeding episodes. In these cases one to three doses of factor are often sufficient to control bleeding, prevent secondary haemorrhage and initiate tissue healing. However, $50-100 \%$ of normal levels are necessary to treat or prevent life-threatening or limb-threatening haemorrhage. In these situations, prolonged hospitalisation for days or weeks may be required ${ }^{4}$. In such cases, factor replacement can be delivered by either intermittent bolus or continuous infusion. When continuous infusion is used, a constant therapeutic factor level is maintained that is not subject to the peaks and troughs observed with bolus injections and total factor use may decrease by as much as $30 \% 6$. The rapid achievement of steady-state plasma factor levels also allows for simplified laboratory monitoring. 


\section{Fresh frozen plasma (FFP)}

The plasma from a donation of whole blood may be siphoned into a satellite bag and frozen. The volume of plasma varies from about $175-250 \mathrm{ml}$, containing about $0.7-0.9 \mathrm{U} / \mathrm{ml}$ of VIII, IX and other plasma clotting factors. Use of whole plasma is limited by the amount of intravascular volume expansion the patient can tolerate ${ }^{1}$. Young patients with normal cardiac and pulmonary function may tolerate as much as $18 \mathrm{ml} / \mathrm{kg}$ given over an hour. Allergic reactions are common in patients who have received plasma often. Treatment with antihistamines is usually effective in controlling such reactions.

If a high level of a clotting factor is needed, FFP can be given in an exchange-plasmapheresis to avoid volume overload. Disadvantages of plasma exchange include its high cost and use of largebore needles ${ }^{1}$.

\section{Cryoprecipitate}

Cryoprecipitate is the most inexpensive factor VIII concentrate available. One bag of cryoprecipitate contains the few $\mathrm{ml}$ of cold-insoluble material remaining after slow thawing of a bag of frozen single-donor plasma. A typical bag, made as a byproduct of whole blood donation contains about 80$100 \mathrm{U}$ of VIII:C and von Willebrand factor, and $200-300 \mathrm{mg}$ of fibrinogen in a volume of $10-20$ $\mathrm{mll}$. One bag of cryoprecipitate $/ 5 \mathrm{~kg}$ of body weight raises the recipient's level to about 50\% (50 $\mathrm{U} / \mathrm{dl}$ ) of normal ${ }^{7}$.

Cryoprecipitate has some disadvantages. It must be transported and stored in the frozen state and thus is less appropriate for home infusion. Allergic reactions may occur; antihistamines usually offer adequate protection or treatment. Furthermore, the potency varies from bag to bag and may be quite low in any one bag ${ }^{1}$.

\section{Lyophilized factor VIII concentrate}

Factor VIII concentrates that are heat-treated or detergent-treated are prepared by monoclonal antibody techniques. These products, as well as genetically engineered or recombinant factor VIII concentrates, are more expensive than cryoprecipitate but are safer with regard to transmission of infectious organisms. They are stable at room temperature. Allergic reactions are rare. They are dispensed as lyophilized powders in bottles of 250-500 units that can be reconstituted just prior to use. They are suitable for self-infusion programmes ${ }^{1}$.

\section{Lyophilized factor IX concentrate}

Low-purity factor IX concentrate contains prothrombin and significant amounts of factors VII, IX, $\mathrm{X}$ and their activated forms and hence is designated as prothrombin complex concentrate (PCC). A typical vial of lyophilized material contains 500-1000 U of factors IX and IXa in 20$30 \mathrm{ml}$ when reconstituted ${ }^{1}$. PCC is stable at refrigerator temperatures and rarely causes allergic reactions and thus is handy for self-infusion programmes $^{1}$. PCC is the mainstay of therapy for severe haemophilia B. It is also widely used to treat haemorrhages in patients with inhibitors to factor VIII.

When PCC is administered at frequent or prolonged intervals, it may cause disseminated intravascular coagulation (DIC) or, paradoxically, thrombosis. Both complications are potentially lifethreatening ${ }^{7}$. High-purity factor IX concentrates have little or no thrombogenic potential but are very expensive ${ }^{8}$.

\section{Treatment at Home}

The early and prompt therapy that is required for the haemophiliac is extremely difficult in the hospital setting. Over the past three decades, an increasing number of severe haemophiliacs have been taught to manage minor haemorrhages with intravenous clotting factor concentrate infusions at home ${ }^{5}$. Patients learn to diagnose their own haemorrhages, to calculate dosage, to perform clean infusions, to keep accurate records and to report to hospital in the event of serious haemorrhages. The paediatrician maintains responsibility for supervision of treatment; therefore, the paediatrician and patient or his parent must remain in telephone contact. Parents may learn to infuse children as young as 2-4 years of age. Boys in early adolescence learn to infuse themselves. Home treatment programmes have met with great success and are ideal for the vast majority of severely affected haemophiliacs ${ }^{5,9}$.

Home treatment allows prompt therapy of incipient haemorrhages so that minimal morbidity ensues. Patients lose far less time from school than if they must travel to a hospital for treatment ${ }^{5,9}$. Levine and Britten (1973) compared the experiences of 45 patients during the year before and the year after introduction of self-infusion and found reductions of $74 \%$ in absence from school, $89 \%$ in days hospitalised, $76 \%$ in outpatient visits and $45 \%$ in total health care $\operatorname{costs}^{10}$. In a later study (Levine, 1974) the incidence of new haemophilic arthropathy was shown to decrease by $86 \%$ on selfinfusion $1^{1}$. Self-infusion also allows the patient and his family to preserve a normal schedule of 
activities most of the time, a benefit that contributes greatly to every member's comfort ${ }^{1}$.

\section{Ancillary therapy}

\section{DDAVP (Desmopressin)}

DDAVP (1-deamino 8-D arginine vasopressin), a synthetic analogue of vasopressin used as replacement therapy in diabetes insipidus, was shown in 1977 to effectively prevent bleeding in patients with mild or moderate haemophilia A or von Willebrand disease ${ }^{12}$. The IV infusion of desmopressin, $0.3 \mathrm{Hg} / \mathrm{kg}$ body weight, typically causes a fourfold rise in the level of factor VIII activity over baseline level. Infused in this way, its peak effect is observed in 30-60 minutes ${ }^{12}$.

Subcutaneous administration of desmopressin has been shown to be as effective as IV administration and can be adapted for self-treatment ${ }^{13}$. Using the present $4 \mu \mathrm{g} / \mathrm{ml}$ formulation, however, patients must inject as much as 4-6 $\mathrm{ml}$ of fluid.

Attempts to develop a more easily administered form of desmopressin for home use have focused on intranasal administration. Initial attempts using a pipette to deliver desmopressin as nasal drops gave erratic and unsatisfactory results ${ }^{14}$. The development of an atomizer that can deliver a measured amount of desmopressin as a nasal spray has made possible better absorption and more reproducible elevation of haemostatic factors. The concentrated DDAVP intranasal spray became available in 1994 and has similar efficacy to the IV preparation in clinical trials, although its peak effect is observed later, at 60-90 minutes after administration $^{15}$. Used in this way, the dose is 150 $\mathrm{Hg}$ (one metered dose) for individuals weighing less than $50 \mathrm{~kg}$ and $300 \mathrm{Hg}$ (two metered doses) for persons weighing $50 \mathrm{~kg}$ or more ${ }^{15}$.

The major side effect of DDAVP is asymptomatic facial flushing. Thrombosis is a rarely reported complication $^{16}$. Because it is synthetic, desmopressin is unlikely to contain infectious agents. Thus, it is the drug of choice for management of patients with mild factor VIII deficiency. Haemostatic levels of VIII:C cannot be maintained with desmopressin alone over many days because of the decreased responsiveness to closely spaced doses. For major surgical operations, desmopressin can be alternated with cryoprecipitate $^{1}$.

\section{Antifibrinolytic therapy}

Antifibrinolytic therapy is used to stabilize a clot by inhibiting the normal process of clot lysis by the fibrinolytic system. These agents provide important adjunctive therapy for the prevention or treatment of oral haemorrhage because saliva is a rich source of fibrinolytic enzymes ${ }^{4}$.

2 drugs are available, epsilon aminocaproic acid (EACA) and tranexamic acid. EACA is administered either orally or IV at an initial dose of $200 \mathrm{mg} / \mathrm{kg}$, followed by $100 \mathrm{mg} / \mathrm{kg}$ every 6 hours (maximum $5 \mathrm{~g} /$ dose) ${ }^{17}$. Tranexamic acid is administered at a dose of $25 \mathrm{mg} / \mathrm{kg}$ (maximum $1.5 \mathrm{~g}$ ) orally, or $10 \mathrm{mg} / \mathrm{kg}$ (maximum $1.0 \mathrm{~g}$ ) IV every 8 hours $^{18}$.

Whether used to treat spontaneous oral haemorrhages or to prevent bleeding from dental procedures in patients with haemophilia A or B, either drug is begun in conjunction with DDAVP or factor-replacement therapy and continued for 2-5 days or until mucosal healing is complete ${ }^{3}$. These drugs are contraindicated in the presence of haematuria, for clots in renal tubules may not be resorbed in the presence of inhibitors of fibrinolysis. They are also usually not given with PCC because of possible enhancement of the thrombotic risk ${ }^{1}$.

\section{Analgesics and anti-inflammatory agents}

Paracetamol with or without codeine is used commonly for chronic pain. Short courses of narcotic analgesics, such as morphine, are sometimes required to relieve the severe pain of acute internal haemorrhages. However, it must be stressed that the best remedy for a painful joint or muscle is not an analgesic or ice bag but an infusion of the deficient factor. As soon as active haemorrhage is stopped, pain usually ceases within minutes to hours ${ }^{1}$.

Corticosteroids may be used briefly to reduce oedematous swelling around acute haemorrhages or to subdue synovial inflammation. Non-steroidal anti-inflammatory agents often provide notable relief of haemophilic arthritis with little or no impedance of haemostasis ${ }^{19}$. Aspirin is contraindicated because it irreversibly impairs platelet function by inhibiting the enzyme cyclooxygenase $^{20}$.

\section{Management of bleeding into specific sites}

\section{Haemarthrosis}

Haemorrhage may occur anywhere in the body but most commonly develops within the joint cavity. The knees, elbows and ankles are most commonly involved. The large ball-and-socket joints (shoulders and hips) and smaller joints in the extremities are affected less frequently ${ }^{21}$. The initial symptom of haemorrhage within a joint is 
pain, followed hours later by swelling, warmth and decreased mobility owing to muscle spasm ${ }^{21}$.

When acute bleeding does occur, the following interventions are mandatory if sequelae are to be prevented $^{4}$ :

1. Prompt factor infusion with repeated doses, as necessary, to ensure complete healing;

2. In older children, enforced rest or splinting of the affected joint until symptoms resolve; followed by

3. A progressive programme of active stretching and strengthening.

Weight bearing is delayed until swelling of the joint has disappeared and the musculature can support the joint. Between exercise sessions, splints are continued to discourage contracture. If thorough rehabilitation is not given after an acute haemarthrosis and the patient is released with weak muscles, a contracted joint or abnormal gait, he will be susceptible to recurrent joint and muscle haemorrhages $^{1,21}$.

A cycle of repeated haemorrhage can occur into a "target" joint such as the ankle, knee or elbow. Direct exposure to blood causes inflammation and hypertrophy of the synovium with deposition of haemosiderin in phagocytic cells. The formation of vascular synovial villi causes greater vulnerability to haemorrhage. Later the cartilage becomes pitted and eroded. Cysts develop in the subchondral bone and osteophytes appear. The epiphyseal plates overgrow, leading to limb-length discrepancies and knob deformities that limit motion. The joint space narrows as cartilage disappears. In the last stage, the joint space is replaced with fibrous tissue and spontaneous fusion may occur. Chronic pain, loss of mobility and disuse atrophy of adjacent muscles is common. This condition is known as haemophilic arthropathy ${ }^{1,21}$.

Chronic synovitis with effusion is occasionally seen in children and adolescents, most commonly involving the knee. The joint is swollen, boggy and warm, but not particularly painful, and not held in the flexed position so characteristic of acute haemorrhage ${ }^{1}$.

At the first sign of a developing target joint, aggressive therapy is initiated. This includes the prophylactic infusion of clotting factor on a 2-4 times weekly schedule to maintain levels of factor VIII or IX above $1-2 \%$ for a period of several weeks to months.
A short 5-7 day course of $1 \mathrm{mg} / \mathrm{kg} /$ day of oral prednisolone may be indicated for patients with severe synovitis. A rehabilitation programme supervised by a physiotherapist and an orthopaedic surgeon is an important part of this regimen ${ }^{4,21}$.

Surgical intervention is rarely necessary. Aspiration of a distended joint is nearly always contraindicated. Enthusiasm for this procedure has waned with the appreciation that the risks of aspiration are not balanced by demonstrable improvement in joint function following removal of blood from the joint cavity ${ }^{21}$. If synovitis persists or haemorrhage recurs despite these conservative measures, arthroscopic or isotopic synovectomy may be indicated to arrest inflammation or bleeding ${ }^{22,23}$.

Arthroscopic synovectomy has largely replaced the open-joint procedure and has yielded good clinical results through the debridement of inflamed synovium $^{22}$. Preoperatively, hospitalisation and high-dose clotting-factor regimens are required for at least 1 week. Subsequently, 6-8 weeks of intensive physiotherapy and prophylactic clotting factors are necessary, followed by rehabilitation for as long as 1 year $^{22}$.

Recently, isotopic synovectomy, or synoviorthesis, was shown to be an efficacious and cost-effective alternative $^{23}$. Performed in an outpatient setting under local anaesthesia, this procedure uses the intra-articular injection of chromic phosphate (32P) to ablate inflamed synovium. In general, only 1-2 days of clotting factor coverage and a short period of subsequent limb immobilisation are required. Experience to date suggests that this procedure may not be as effective as arthroscopic synovectomy in preventing recurrent haemarthroses but that joint range of motion may be better preserved ${ }^{23}$.

\section{Muscle bleeding}

The second most common location of bleeding is within muscles, particularly the forearm flexor muscle compartment, gastrocnemius and iliopsoas. Nerve compression resulting in permanent injury is a major risk in such instances ${ }^{24}$. The initial symptom of haemorrhage within a muscle is pain followed hours later by swelling, warmth and decreased mobility owing to muscle spasm ${ }^{21}$. Most important in the treatment of such episodes is the immediate replacement of the deficient factor by means of intravenous infusion to a level adequate to stop haemorrhage $e^{5,24}$.

Bleeding into the iliopsoas muscle is responsible for a clinical syndrome that is misdiagnosed as an acute abdomen or hip bleed ${ }^{25}$. The patient presents with pain and tenderness in the groin, usually 
maximal at the mid-inguinal point or femoral ring, and occasionally with discomfort in the scrotum or iliac fossa. The hip is held in a flexed position; extension is painful but gentle rotation is tolerated in contrast to intra-articular hip haemorrhage, in which rotation is exquisitely painful. The femoral nerve is usually compressed with decreased sensation over the anterior thigh and knee area, absence of the knee jerk and weakness of the quadriceps muscle $e^{1,25}$. The presence of an iliopsoas haemorrhage can be confirmed by ultrasound scan or computed tomography. Haemorrhages of the ileopsoas, quadriceps, hamstrings and gastrocnemius muscles require bed rest and repeated plasma product infusion until swelling subsides. Mobilisation is cautious for recurrences are common. Prolonged physiotherapy is often needed to regain strength ${ }^{1,25}$.

\section{Central nervous system (CNS)}

CNS bleeding is the most common cause of death in haemophilia ${ }^{26}$. An injury that is usually trivial in a normal person, such as knocking the head against a cabinet while standing up or falling on the buttocks and jolting the vertebral column, may lead to prolonged bleeding from a small CNS vessel in a haemophiliac ${ }^{1}$. The slowly accumulating blood eventually causes symptoms after a few hours or days. Many patients with CNS bleeding cannot recall an injury. Any patient with symptoms referable to the spinal cord or brain, including unusual headache or lethargy, should be hospitalised, treated with the appropriate plasma product to maintain factor VIII levels of $50 \mathrm{U} / \mathrm{dl}$ or more, or factor IX levels of $40 \mathrm{U} / \mathrm{dl}$, and observed closely ${ }^{1}$.

\section{Mouth, pharynx and neck}

Bleeding in the mouth from lacerations or a bitten tongue is often persistent. Blood loss in a small child may be excessive; red cell replacement may be needed. A large friable clot, resembling a raspberry, may protrude from the wound, holding the edges apart. Such clots must be wiped off when the appropriate plasma product infusion is given so that the wound edges may come together. EACA or tranexamic acid is useful in preserving clots in the mouth $^{1}$.

Bleeding under the tongue, behind the pharyngeal wall, or in the neck is dangerous because it may choke the patient. Hospitalisation and maintenance of plasma VIII levels of $50 \mathrm{U} / \mathrm{dl}$ or IX levels of 30 $40 \mathrm{U} / \mathrm{dl}$ are appropriate. Haemorrhages in these areas may be brought on by tonsillitis, by spells of coughing or by regional block anaesthesia for dentistry ${ }^{1}$.

\section{Haematuria}

Haematuria is fairly common in severe haemophilia. Clots sometimes obstruct renal tubules or the ureter, causing temporary renal colic and, sometimes, hydronephrosis ${ }^{1}$. A high fluid intake may dilute the blood in the tubules and ureter and prevent formation of large clots. Clinicians disagree on the value of plasma product infusion. Some feel that plasma products are appropriate. Others, noting that haematuria often persists despite plasma product administration, and that clots may cause obstruction, prefer not to use plasma products. The immobilisation provided by bed rest is often helpful. EACA and tranexamic acid should not be administered to patients with haematuria ${ }^{1}$.

\section{Dental management}

Regular dental care should be encouraged. Cleaning and scaling of the teeth may cause mild oozing but plasma products are rarely needed. The major problem in the restoration of carious teeth is the choice of anaesthetic. Local infiltrative anaesthetics can be used without prior plasma product infusion but elevation of the plasma factor VIII or IX level to $30-40 \mathrm{U} / \mathrm{dl}$ is advisable before regional block anaesthetic. Although most such anaesthetics given to haemophiliacs cause no harm, occasionally the needle pierces a large vessel, causing a rapidly enlarging haematoma at the side of the jaw that may dissect into the neck and compress the trachea ${ }^{1}$.

Most dental extractions are managed easily without hospitalisation. The plasma level of factor VIII or IX is raised immediately before the extraction. EACA or tranexamic acid is usually given on the day of extraction and for about 7-10 days afterwards. A strict diet of cold liquids for several days helps to preserve clots. Plasma products are seldom necessary in the postoperative period ${ }^{1}$.

\section{Surgical operations}

Surgical operations on haemophiliacs should take place at haemophilia centres where appropriate care can be given. The surgeon should be aware of the special needs of the haemophiliac and should maintain close contact with the paediatrician and the haematologist. An ample supply of the appropriate blood product should be on hand ${ }^{1}$.

Before the procedure, the patient should have a sensitive test to rule out the presence of an inhibitor. About an hour before the operation, the appropriate plasma product is given to raise the plasma VIII level above $50 \mathrm{U} / \mathrm{dl}$ or the factor IX level to $40 \mathrm{U} / \mathrm{dl}$. After infusion, the plasma clotting 
factor level is checked with a specific factor assay. Additional plasma products may be given during a lengthy operation. In the post-operative period, the clotting factor level is maintained at a minimum of 40-50 VIII U/dl or 30-40 IX U/dl for 10-14 days. Specific factor assays are used to make sure that clotting factor levels are adequate. Most patients are hospitalised for at least 10-14 days and often longer if an orthopaedic procedure is followed by intensive physiotherapy ${ }^{1}$.

\section{Prophylactic factor replacement}

Recurrent joint bleeding followed by the development of target joints, chronic synovitis and chronic arthropathy may lead to severe physical impairment, loss of time from school, psychosocial consequences and increased long-term disability. Therefore, prophylaxis against haemarthroses and subsequent irreversible joint injury should be the goal of therapy for patients with haemophilia. Based on earlier observations that persons with moderate haemophilia rarely develop chronic arthropathy, it has been theorized that adequate prevention could be accomplished by maintaining a minimum factor VIII or IX level of $1-5 \%{ }^{24}$.

Bleeding prophylaxis has been practised in Europe over the past 20 years. The Swedish experience has included the institution of bleeding prevention at age 1-3 years before significant bleeding begins ${ }^{27}$. In their experience, 21 young children, treated with $25-40 \mathrm{U} / \mathrm{kg}$ of factor VIII thrice weekly or similar doses of factor IX twice weekly, had fewer than one haemarthrosis per year and normal joints by standardised orthopaedic and radiologic evaluations over 10 years $^{28}$. These children led normal lives both physically and socially; however, their mean yearly factor VIII or IX usage was 6000 $\mathrm{U} / \mathrm{kg} /$ year, a more than fourfold increase over the on-demand factor usage for patients with severe haemophilia.

Conversely, the European experience also suggested that secondary prophylaxis, instituted at a somewhat later age because of previous significant joint bleeding and early arthropathy, dramatically decreased but did not abolish joint haemorrhage ${ }^{29,30}$. Although both quality of life and orthopaedic examinations improved on these regimens, the degree of joint damage progressed radiographically in many cases ${ }^{29,30}$. Therefore, despite a two-to-threefold increase in factor usage, long-term orthopaedic disability could not be successfully averted when prophylaxis was initiated after significant joint haemorrhage had occurred. Secondary prophylaxis performed in the United States also has confirmed European experience, with evidence of eventual radiographic progression of joint disease despite clinical improvement $^{31}$.

\section{Inhibitor development and therapy}

One of the most life-threatening complications of haemophilia and its therapy is the development of polyclonal antibodies to factor VIII or IX that neutralise or "inhibit" factor coagulant activity, rendering specific replacement therapy less or not at all effective ${ }^{32}$. They are, therefore, referred to as inhibitors.

An inhibitor is usually detected in one of two ways ${ }^{4}$ :

1. It may be discovered in an asymptomatic individual during routine screening performed once or twice yearly, or

2. It may be detected by the abrupt onset of clinical unresponsiveness to usual or higher doses of clotting factor.

The presence of an inhibitor is confirmed by the Bethesda inhibitor assay, a clot-based assay that titres the amount of neutralising antibody present in plasma on the basis of its ability to inhibit factor coagulant activity in vitro. This measurement is reported in "Bethesda Units" (BU), with higher Bethesda titres reflecting greater amounts of inhibiting antibody ${ }^{4}$.

Haemophiliacs who have antibodies are classified as having "high-responding" or "low-responding" inhibitors. High responders have brisk anamnestic responses with repeat exposures to specific factor therapy. They consequently develop high titre antibodies that may or may not fall to undetectable levels in the absence of further factor exposure over months to years. On the other hand, low responders have minimal to no anamnesis on repeat exposure to factor VIII or IX and their Bethesda titres generally remain low. These inhibitor characteristics can alter with time ${ }^{4}$.

Inhibitors occur in $20-33 \%$ of persons with haemophilia $\mathrm{A}^{32}$ but in only $1-4 \%$ of persons with haemophilia $\mathrm{B}^{33}$. Most of these antibodies develop during childhood. $80 \%$ of factor VIII antibodies were of the high-responding type; transient inhibitors were observed uncommonly ${ }^{4}$. With the exclusive use of recombinant factor VIII products, less than one half were high responding and a considerable number were transient ${ }^{34}$. Similar information on the nature of factor IX inhibitors is less available because of their low prevalence. One survey in the United States identified 11 of 29 $(38 \%)$ factor IX inhibitor patients as having 
inhibitor titres of $5 \mathrm{BU}$ or less, with the remainder having higher titre inhibitors ${ }^{35}$.

The pathophysiology of this antibody development is not yet clear. Clues may lie in the observation that inhibitor development is more common among more severe forms of haemophilia A, members of the same family and black individuals ${ }^{36}$.

Therapy for bleeding in the presence of an inhibitor can be challenging. In an individual with a lowresponding inhibitor and a low Bethesda titre, control of both minor and major haemorrhage is frequently possible with factor VIII or IX replacement therapy. Higher doses and more frequent or continuous factor infusion may be necessary to achieve haemostatic factor levels. This therapeutic regimen can also be applied to the treatment of life-threatening or limb-threatening bleeding in a person with a high-responding inhibitor whose Bethesda titre is low; however, a Bethesda titre of $10 \mathrm{BU}$ generally precludes even high-dose specific factor therapy ${ }^{4}$.

Occasionally, the temporary removal of antibody by plasmapheresis can allow the short-term use of specific factor replacement for life-threatening or limb-threatening haemorrhage ${ }^{37}$.

For persons with haemophilia A, the use of porcine factor VIII is another treatment option. Given its significant homology with human factor VIII, the porcine product can effectively participate in human coagulation physiology while remaining undetectable by a portion of the antifactor VIII antibodies in many haemophilia A patients with inhibitors $^{38}$. Porcine factor VIII is used primarily for major haemorrhage, given its associated allergic and haematologic side effects and its propensity to cause an anamnestic rise in antibodies to both porcine and human factor VIII. This product may be less effective when porcine inhibitor titres are greater than $15-20 \mathrm{BU}^{38}$.

In haemophilia B and frequently in haemophilia $\mathrm{A}$, the treatment of both minor and major haemorrhage consists of the use of PCC or activated prothrombin complex concentrates (APCC). PCC and APCC are known as bypass therapy since these products bypass the requirement for factor VIII or IX for clot formation. APCC differ from PCC in that they undergo a manufacturing process that increases their content of activated factors II, VII, IX and X. Consequently, the cost of APCC is fourfold higher than that of PCC. Increased efficacy of APCC as compared with PCC has not been well documented by controlled clinical trials. Used in the treatment of inhibitor bleeding since the 1970s, PCC and APCC are effective but less so than specific factor therapy ${ }^{39}$. Although this therapy is short acting, its frequent use has significant drawbacks. Furthermore, because they contain small amounts of factor VIII and larger quantities of factor IX, these products also can cause anamnesis in highresponding haemophilia $\mathrm{A}$ or $\mathrm{B}$ inhibitor patients ${ }^{40}$.

Recombinant factor VIIa, a new genetically engineered clotting factor preparation, is undergoing prelicensure clinical trials. Although preliminarily shown to be effective in the treatment of central nervous system and other bleeding, more clinical experience obtained through carefully controlled clinical trials is necessary to assess its overall safety and efficacy ${ }^{41,42}$.

None of this alternative treatment for haemophilic bleeding in the presence of an inhibitor ensures the same satisfactory outcome as specific factor replacement therapy in children without inhibitors. Consequently, persons with inhibitors suffer more life-threatening complications from their disease and have greater short-term and long-term disability. Therefore, inhibitor eradication is the best long-term option for these patients. This can only be accomplished through a process called immune tolerance, the goal of which is to render the individual immunologically tolerant to the deficient clotting factor. This therapy requires the regular (daily to weekly) infusions of factor VIII or IX by either bolus injection or continuous infusion for a period of weeks to years, with or without the concomitant use of immunomodulatory therapy, such as low-dose cyclophosphamide, high-dose gamma globulin or prednisolone $\mathrm{s}^{43,44}$. Although this process has an overall $60-80 \%$ success rate, it is time-consuming, associated with central venous access complications and often limited by its prohibitive cost. Immune tolerance may be most applicable, successful and cost effective, however, when performed in paediatric patients as soon as possible after inhibitor development ${ }^{43,44}$.

\section{Hepatitis $C$ and chronic liver disease}

$60-95 \%$ of haemophiliacs more than 7 or 8 years of age have been infected with hepatitis $\mathrm{C}$ virus (HCV). In one survey, $98 \%$ of HIV-infected persons with haemophilia were HCV-positive, whereas $80 \%$ of HIV-negative patients were similarly HCV-positive ${ }^{45}$. Although $70-80 \%$ of acute HCV infection may be asymptomatic, longterm sequelae are noted in at least $85 \%$ of exposed persons. Chronic hepatitis $\mathrm{C}$ is considered an indolent infection, with asymptomatic periods ranging from 14-23 years ${ }^{46}$.

Unfortunately, treatment studies in nonhaemophilic patients with interferon alpha $2 \mathrm{~b}$ (IFN) have to date yielded only a $25 \%$ sustained remission rate ${ }^{47}$. 
Combined studies on a total of 57 haemophiliac patients have yielded a lower overall sustained response rate of $12 \%$ and $\mathrm{HIV}$-infected patients yielded particularly poor results ${ }^{48}$. Pilot studies using a combination of ribavirin and IFN have been encouraging ${ }^{49}$. The current therapeutic dilemma is that the best responders to this therapy are those $\mathrm{HCV}$-infected individuals who have minimal clinical, biochemical and histologic disease and who may not necessarily need treatment ${ }^{4}$.

\section{Carrier detection and genetic counselling}

Since haemophilia A and B are X-linked recessive disorders, it would be expected that the patients' mothers are carriers. This is certainly the case when there are 2 affected sons, one son and other male relatives with haemophilia, or a father with the disease. On the other hand, a woman is considered to be a possible carrier when she has only a single haemophilic son without a family history or when she is a sister or more distant relative of a haemophiliac. For such an individual, several options are available to accurately define the risk of having affected offspring ${ }^{5}$.

Low factor VIII clotting activity (FVIII:C) is present in only $25-40 \%$ of carrier women because of the wide normal range of factor VIII and random inactivation of the $\mathrm{X}$ chromosome (Lyon hypothesis $)^{5}$. In carriers there will usually be twice as much factor VIII-related antigen (FVIIIR:Ag) compared with FVIII:C. Thus, simultaneous measurement of FVIII:C and FVIIIR:Ag and statistical analysis of the results will permit identification of $80-90 \%$ of carriers ${ }^{50}$. Such laboratory analysis supplements calculation of risk according to standard mendelian genetics ${ }^{51}$. 60$70 \%$ of haemophilia B carriers can be detected by the measurement of reduced plasma factor IX activity $^{52}$.

Currently, carrier detection in haemophilia A can be performed using intragenic and extragenic linkage analysis of DNA polymorphisms ${ }^{53}$. These gene-tracking techniques are useful with up to $99.9 \%$ precision but only when an affected male patient and his intervening family members are available for analysis ${ }^{53}$. Direct gene-mutation analysis for the intron 22 inversion is recommended for carrier detection in severe haemophilia A families when affected and intervening family members are unavailable ${ }^{54}$. Direct gene-mutation analysis is currently available in haemophila B carrier detection ${ }^{55}$.

\section{Gene therapy}

After factor VIII and IX genes were cloned, haemophilia A and B have been considered to be diseases that are most amenable to gene therapy because of 3 reasons ${ }^{56}$ :

1. Lack of requirement for tissue-specific expression.

2. Absence of precise regulation of these factor proteins.

3. Clinical benefit derived from less-than-normal factor expression and, conversely, the lack of known adverse effect from protein overexpression..

The goal of gene therapy is the safe continuous delivery of sufficient amounts of factor VIII or IX to prevent haemorrhagic symptoms ${ }^{56}$. This has been achieved by in vivo or ex vivo addition of factor VIII or IX gene to target cells using viral vectors for gene transfer. Retroviruses, adenoviruses and adeno-associated viruses have been the vectors most used ${ }^{56}$.

The factor IX gene has so far been the most amenable to transfer, given the smaller size of its cDNA $(3 \mathrm{~kb})$ and the larger physiologic volume of distribution of the factor IX protein, compared with factor VIII. The factor VIII gene has been more problematic because of its larger size $(9 \mathrm{~kb})^{56}$.

\section{Sri Lankan situation}

In 1954, the Medical Research Council of the United Kingdom designated certain clinics as haemophilia centres to diagnose, register and treat haemophiliac patients ${ }^{57}$. In 1964, a prototype comprehensive rehabilitation project for haemophilia was established in Los Angeles by the Vocational Rehabilitation Administration of the United States of America ${ }^{58}$. However, even in 2002, there is not a single haemophilia centre established in Sri Lanka. It is about time that haemophilia centres are established in the major teaching hospitals in Colombo, Kandy, Galle and Jaffna.

Home therapy, which is a feature of haemophilia management throughout the world, is not practised here. This may be because of the difficulty of teaching our mothers to give IV clotting factor infusions at home. However, a concentrated DDAVP intranasal spray is available which can be used at home. $150 \mu \mathrm{g}$ (one-metered dose) can be given to persons weighing less than $50 \mathrm{~kg}$, at the onset of haemorrhage, before bringing the child to hospital. By doing this, joint bleeds can be prevented to a large extent. The only problem may be the cost of the DDAVP nasal spray.

Neither primary nor secondary bleeding prophylaxis is practised in Sri Lanka. Bleeding 
prophylaxis consists of giving the haemophiliac children $25-40 \mathrm{U} / \mathrm{Kg}$ of factor VIII thrice weekly or factor IX twice weekly ${ }^{4}$. Primary prophylaxis is started at 1-3 years of age before significant bleeding begins. Secondary prophylaxis is started at a somewhat later age because of previous significant joint bleeding and early arthropathy ${ }^{4}$. Prophylaxis, especially secondary prophylaxis, is feasible in Sri Lanka.

Prothrombin complex concentrate (PCC), which is the mainstay for treatment of major bleeding episodes in haemophilia B, is not available in Sri Lanka. This is hard to understand because the cost of PCC must be about the same as the cost of factor VIII concentrate. Furthermore, PCC is effective in treatment of inhibitor bleeding.

DDAVP should be made available in government hospitals as it is the ideal treatment for mild haemophilia A and being synthetic, is unlikely to contain infectious agents.

Inhibitor assessment is done qualitatively rather than quantitatively in Sri Lanka. Thus we can only know whether haemophiliacs are having inhibiting antibody but not whether the antibodies are "highresponding" or "low-responding". Whilst higher doses and more frequent or continuous factor infusion can achieve haemostatic factor levels when the inhibitor level is low, a Bethesda titre of $10 \mathrm{BU}$ precludes even high-dose specific factor therapy. These are the cases where PCC may be effective.

Factors VIII and IX assays are now available in Sri Lanka and hence carrier detection can be carried out.

\section{Recommendations}

In conclusion, I wish to make the following recommendations:

- Establish haemophilia centres in the main teaching hospitals.

- Make PCC available in Sri Lanka.

- Start home therapy using the concentrated DDAVP intranasal spray.

- Make DDAVP available in hospitals.

- Start primary or secondary bleeding prophylaxis.

- Make quantitative inhibitor assays available in Sri Lanka.

\section{References}

1. Kasper C K, Dietrich S L. Comprehensive management of haemophilia. Clinics in Haematology 1985; 14: 489-512.

2. Agle D P, Clements M, Mattson A. The prevention of psychological and social disorders in haemophilic care. In: Abe T, De Vreker $\mathrm{R} A$, Nagas $\mathrm{T}$ et al. editors. Proceedings of the Second International Symposium on Haemophilia Treatment. Tokyo; Kyoritsu Printings 1981; 115-120.

3. Green W B, Strickler E M. A modified isokinetic strenghening program for patients with severe haemophilia. Developmental Medical Child Neurology 1983; 25: 189-96.

4. Di Michele D. Haemophilia 1996; new approach to an old disease. Paediatric Clinics of North America 1996; 43: 709-36.

5. Buchanan G R. Haemophilia. Paediatric Clinics of North America 1980; 27: 309-26.

6. Bona R D, Weinstein R A, Weisman S J et al. The use of continuous infusion of factor concentrates in the treatment of haemophilia. Am J Hematol 1989; 32: 8-13.

7. Lusher J M. Prediction and management of adverse events associated with the use of factor IX complex concentrates. Semin Hematol 1993; 30: 36-40.

8. Kim H C, McMillan C W, White $\mathrm{G} \mathrm{C}$ et al. Purified factor IX using monoclonal immunoaffinity technique: Clinical trials in haemophilia $\mathrm{B}$ and comparison with prothrombin complex concentrates. Blood 1992; 79: 568-72.

9. Agle D P, Hilgartner M W, Lazerson J et al. Home therapy for haemophilia: A physician's manual. New York; The National Haemophilia Foundation, 1977.

10. Levine P H, Britten A F H. Supervised-patient management of haemophilia. A study of 45 patients with haemophilia A and B. Annals of Internal Medicine 1973; 78: 195-201.

11. Levine $P$ H. Efficacy of self-therapy in haemophilia: A study of 72 patients with haemophilia A and B. New England Journal of Medicine 1974; 291: 1381-4. 
12. Manucci P M, Pareti F I, Ruggeri Z M et al: 1Deamino-8-D-arginine vasopressin: A new pharmacological approach to the management of haemophilia and von Willebrand's disease. Lancet 1977; 1: 869-72.

13. Mannucci P M, Vicente V, Alberca I et al Intravenous and subcutaneous administration of desmopressin (DDAVP) to haemophiliacs: pharmacokinetics and factor VIII responses. Thromb Haemost 1987; 58: 1037-9.

14. Kohler M, Hellstern P, Miyashita C, von Blohn G, Wenzel E. Comparative study of intranasal, subcutaneous and intravenous administration of desamino-Darginine vasopressin (DDAVP). Thromb Haemost 1986; 55: 108-11.

15. Rose E H, Aledort L M. Nasal spray desmopressin (DDAVP) for mild haemophilia $\mathrm{A}$ and von Willebrand disease. Ann Intern Med 1991; 114: 563-8.

16. Mannucci P M, Lusher J M. Desmopressin and thrombosis. Lancet 1989; 2: 657-76.

17. Corrigan J J. Oral bleeding in haemophilia: Treatment with epsilon aminocaproic acid and replacement therapy. J Pediatr 1972; 80: 1248.

18. Tavenner $\mathrm{R} \mathrm{W}$ H. Use of tranexamic acid in control of haemorrhage after extraction of teeth in haemophilia and Christmas disease. British Medical Journal 1972; ii: 314-5.

19. Thomas P, Hepburn B, Kim H C, Saidi P. Nonsteroidal anti-inflammatory drugs in the treatment of haemophilc arthropathy. American Journal of Haematology 1982; 12: 131-7.

20. Kaneshiro M M, Mielke C H, Kasper C K, Rapaport S I. Bleeding times after aspirin in disorders of intrinsic clotting. New England Journal of Medicine 1969; 281: 1039-42.

21. Arnold W D, Hilgartner M W. Haemophiliac arthropathy. Current concepts of pathogenesis and management. J Bone Joint Surg 1977; 59A: 287-305.

22. Kim $\mathrm{H} \mathrm{C}$, Klein $\mathrm{K}$, Hirsch $\mathrm{S}$ et al. Arthroscopic synovectomy in the treatment of haemophilic synovitis. Scand $J$ Haematol 1984; 33: 271-9.
23. Rivard G E, Girard M, Belanger $\mathrm{R}$ et al. Synoviorthesis with colloid 32P chromic phosphate for the treatment of haemophilic arthropathy. J Bone Joint Surg 1994; 76-A: 482-8.

24. Ahlberg A. Haemophilia in Sweden. Incidence, treatment and prophylaxis of arthropathy and other musculoskeletal manifestations of haemophilia A and B. Acta Orthop Scand 1965; 77: 7-80.

25. Goodfellow J, Fearn C B, Mathews J M. Iliacus haematoma. A common complication of haemophilia. J Bone Joint Surg 1967; 49B: 748-56.

26. Biggs R. Haemophilia treatment in the United Kingdom from 1969 to 1974. British Journal of Haematology 1977; 35: 487-504.

27. Nilsson I M, Berntorp E, Lofquist $\mathrm{T}$ et al. Twenty-five years' experience of prophy-lactic treatment in severe haemophilia A and B. $J$ Intern Med 1992; 232: 25-32.

28. Pettersson H, Ahlbert A, Nilsson I M. A radiologic classification of haemophilia arthropathy. Clin Orthop 1980; 149: 153-9.

29. Schramm W. Experience with prophylaxis in Germany. Semin Hematol 1993; 30: 12-5.

30. van den Berg $H$ M, Nieuwenhius $H \quad K$, Mauser-Bunschoten E P. Haemophilia prophylaxis in the Netherlands. Semin Hematol 1994; 31: 13-5.

31. Manco-Johnson M J, Nuss R, Geraghty S et al. A prophylactic program in the United States: Experience and issues. Semin Hematol 1994; 31:10-2.

32. Ehrenforth S, Kreutz W, Scharrer I et al. Incidence of development of factor VIII and factor IX inhibitors in haemophiliacs. Lancet 1992; 339: 594-8.

33. Briet E, Reisner H M, Roberts H R. Inhibitors in Christmas disease. Prog Clin Biol Res 1984; 150: $123-9$.

34. Bray G L, Gomperts E D, Courter S et al. A multicentre study of recombinant factor VIII (Recombinate): Safety, efficacy and inhibitor risk in previously untreated patients with haemophilia A. Blood 1994; 83: 2428-35. 
35. Katz J. Factor IX inhibitors in patients with haemophilia B. Survey results from 82 treatment centres in the United States and Canada. Blood 1994; 84: 63.

36. Gill P M, Shapiro S S, Poole W K et al. The natural history of factor VIII antibodies in patients with haemophilia A. Prog Clin Biol Res 1984; 150: 19-29.

37. Slocombe G W, Newland A C, Colvin M P et al. The role of intensive plasma exchange in the prevention and management of haemorrhage in patients with inhibitors to factor VIII. $\mathrm{Br} J$ Haematol 1981; 47: 577-87.

38. Brettler D B, Forsberg A D, Levine P H et al. The use of porcine factor VIII concentrate (Hyate:C) in the treatment of patients with inhibitor antibodies to factor VIII: A multicentre US experience. Arch Intern Med 1989; 149: 1381-5.

39. Lusher J M, Shapiro S S, Palascak J E et al. Efficacy of prothrombin complex concentrate in haemophiliacs with antibodies to factor VIII. $N$ Engl J Med 1980; 303: 421-5.

40. Yoshioka A, Nakagawa O, Uehara Y et al. In vitro characterisation of various heat-treated prothrombin complex concentrates (PCC). Thromb Res 1987; 46: 827-36.

41. Rice $\mathrm{K}$ M, Savidge $G$ F. NovoSeven (recombinant factor VIIa) in central nervous system bleeds. Haemostasis 1996; 26: 131-4.

42. Bech R M. Recombinant factor VIIa in joint and muscle bleeding episodes. Haemostasis 1996; 26: $135-8$.

43. Kreutz W, Ehrenforth S, Funk M et al. Immune tolerance therapy in paediatric haemophilias with factor VIII inhibitors: 14 years' follow-up. Haemophilia 1995; 1: 24-32.

44. Nilsson I M, Berntorp E, Zettervall O. Induction of immune tolerance in patients with haemophilia and antibodies to factor VIII by combined treatment with intravenous IgG, cyclophosphamide and factor VIII. $N$ Engl J Med 1988; 318: 947-50.

45. Troisi C L, Hollinger F B, Hoots W K et al. A multicentre study of viral hepatitis in a United States haemophilic population. Blood 1993; 81: 412-8.

46. Kiyosawa K, Akahane Y, Nagata A et al. Significance of blood transfusion in non-A non-B chronic liver disease in Japan. Vox Sang 1982; 43: 45-52.
47. Davis G L, Balart L A, Schiff E R et al. Treatment of chronic hepatitis $\mathrm{C}$ with recombinant interferon alpha: A multicentre, randomised, controlled trial. Hepatitis Interventional Therapy Group. $N$ Engl J Med 1989; 321: 1501-6.

48. Makris M, Preston F E, Triger D R et al. A randomised controlled trial of recombinant interferon-alpha in chronic hepatitis $\mathrm{C}$ in haemophiliacs. Blood 1991; 78: 1672-7.

49. Brillanti S, Garson J, Foli M et al. A pilot study of combination therapy with ribavirin plus interferon alpha for interferon alpha-resistant chronic hepatitis C. Gastroenterology 1994; 107: 812-7.

50. Klein H G, Aledort L M, Bouma B N et al. A cooperative study for the detection of the carrier state of classic haemophilia. $N$ Engl J Med 1977; 296: 959-62.

51. Graham J B. Genetic counselling in classic haemophilia A. N Engl J Med 1977; 296: 996-7.

52. Graham J B. Genotype assignment (carrier detection) in the haemophilias. Clin Hematol 1978; 8: 115-45.

53. Janco R L, Philips J A, Orlando $\mathrm{P} J$ et al. Detection of haemophilia A carriers using intragenic factor VIII:cDNA polymorphisms. Blood 1987; 69: 1539-41.

54. Jenkins $\mathrm{P}$ V, Collins $\mathrm{P}$ W, Goldman $\mathrm{E}$ et al. Analysis of intron 22 inversions of the factor VIII gene in severe haemophilia A: Implications for genetic counselling. Blood 1994; 84: 2197-201.

55. Bottema C D K, Koeberl D D, Sommer S S. Direct carrier testing in 14 families with haemophilia B. Lancet 1989; 2: 526-9.

56. Lozier J N, Brinkhous K M. Gene therapy and the haemophilias. JAMA 1994; 271: 47-51.

57. Biggs R, Spooner R J D. National survey of haemophilia and Christmas disease patients in the United Kingdom: Report on behalf of the haemophilia reference centre directors of the UK. Lancet 1978; I: 1143-4.

58. Andonian A A, Dietrich S L, Whiteman S T, Boone D C, Hurt C. A total programme for the patient with haemophilia. Journal of the American Physical Therapy Association 1966; 46: $1268-85$. 